\title{
NUCLEI OF STRAIN AT THREE-DIMENSIONAL BIMATERIAL INTERFACES
}

\author{
$\mathrm{BY}$ \\ X. MARKENSCOFF AND W. YE \\ University of California at San Diego, La Jolla, CA
}

\begin{abstract}
When nuclei of strain approach the interface of two materials, the displacement fields may not be unique and may depend on the direction from which the interface is approached. For example, the displacement fields of a center of dilatation at the interface of two materials are not unique and depend on the direction of approach to the interface. To avoid misunderstanding, it can be stressed that each of the two fields is continuous at the interface. In this paper, we show that there are 12 independent displacement functions of second-order singularities uniquely defined at an interface. The limits of all other nuclei of strain at the interface are linear combinations of these 12 independent displacement functions.
\end{abstract}

I. Introduction. The Kelvin solution for the stress field of a point force in a threedimensional infinite isotropic solid gives a fundamental result in the linear theory of elasticity. By differentiation of this solution, a family of nuclei of strain or force doublets can be obtained. The Galerkin vectors for the complete set of 40 physically significant nuclei of strain in the half-space were given by Mindlin and Cheng (1950). By using Papkovitch functions, Rongved (1955) gave the elastic solutions for a point force in joined half-spaces of different elastic properties for the case when the half-spaces are perfectly bonded to each other. Dundurs and Hetényi (1965) derived the analogous solutions for a sliding contact interface. In 1987, Vijayakumar and Cormack presented a comprehensive formulation of Green's functions for biharmonic equations with respect to perfectly bonded, bimaterial, linear, isotropic, elastic media. They also presented the fundamental solutions for bimaterial elastic media with sliding interface by using the nuclei of strain method. Yu and Sanday (1991) presented the elastic solutions for the complete set of 40 physically significant nuclei of strain in two joined elastic half-spaces of different elastic properties. One year later, Carvalho and Curran (1992) provided twodimensional plane-strain fundamental solutions for elastic bimaterials using the nuclei of strain method. The method is a reduction of the three-dimensional approach previously derived by Vijayakumar and Cormack.

Received April 24, 1995.

1991 Mathematics Subject Classification. Primary 73C02.

(C)1998 Brown University 
We verified here that when the nuclei of strain approach the interface of two materials, the elastic solutions may not be unique: the limits of the stress fields when the nuclei of strain approach the interface either from one material or from the other material may be different. Of course, each one of them has continuous tractions at the interface. For example, the displacement field (continuous at the interface) of a center of dilatation is not unique as the center of dilatation approaches the interface, and it depends on the direction of approach. So it is important to find the fields that are unique at the interface.

In two dimensions, Markenscoff and Dundurs have already found four independent fields that are uniquely defined at the interface between two half-spaces (Markenscoff, Dundurs, and Ye, 1992). They are two force doublet fields and two dislocation-dipole fields. All the other limits of second-order singular fields (in the stress) as they approach the interface are linear combinations of these four fields.

When $\mathrm{Yu}$ and Sanday (1991) presented the fields of the nuclei of strain in two joint half-spaces, they did not consider the limits when they approach the interface. Here, the displacement fields for nuclei of strain applied at the interface of two perfectly joined elastic half-spaces of different elastic properties are presented. The method for obtaining the uniquely defined nuclei of strain at the interface consists of considering the displacement fields due to the forces $P_{x}, P_{y}, P_{z}$ applied at the interface, which are unique, and taking their derivatives $\frac{\partial}{\partial x}, \frac{\partial}{\partial y}$ on the plane of the interface. Because of additional symmetries among these displacement gradients, the number of the independent displacement functions of the nuclei of strain is 12 . We have thus obtained 12 independent displacement functions that are uniquely defined at the interface. The limits of all other displacement fields due to nuclei of strain approaching the interface from either side are observed to be linear combinations of these 12 independent displacement functions.

II. Uniquely defined nuclei of strain at a 3-D interface. In two dimensions the uniquely determined second-order fields at an interface have been obtained by Markenscoff, Dundurs, and Ye (1992) by considering the concentrated forces $P_{x}, P_{y}$ which give unique stress fields, and taking derivatives along the interface axis.

For three dimensions, we proceed to obtain the uniquely determined nuclei of strain at an interface by an analogous approach. We start by considering the concentrated forces $P_{x}, P_{y}, P_{z}$ at an interface (Rongved, 1955) which give unique fields for a bonded interface. (We may note that for a slipping interface these are not unique but depend on the direction of approach (Dundurs and Hetényi, 1965).)

There are three concentrated forces $P_{x}, P_{y}, P_{z}$ that are first-order singular fields in the displacement uniquely defined at the interface of two materials in three dimensions (see Fig. 1). By taking the derivatives of the displacement fields due to the forces $P_{x}, P_{y}$, $P_{z}$ with $x, y, z$, the second-order singularities in the displacement (force doublets) are obtained. Among all these force doublets, those that are obtained by taking the derivatives of the displacement fields due to the forces $P_{x}, P_{y}, P_{z}$ with respect to $z$ (the axis perpendicular to the interface plane) are not uniquely defined at the interface. The limits of these force doublets approaching the interface from materials I and II are different. However, the ones that are obtained by taking the derivatives along the interface, i.e., the derivatives $\frac{\partial}{\partial x}, \frac{\partial}{\partial y}$, are uniquely defined at the interface of two materials. Thus we 


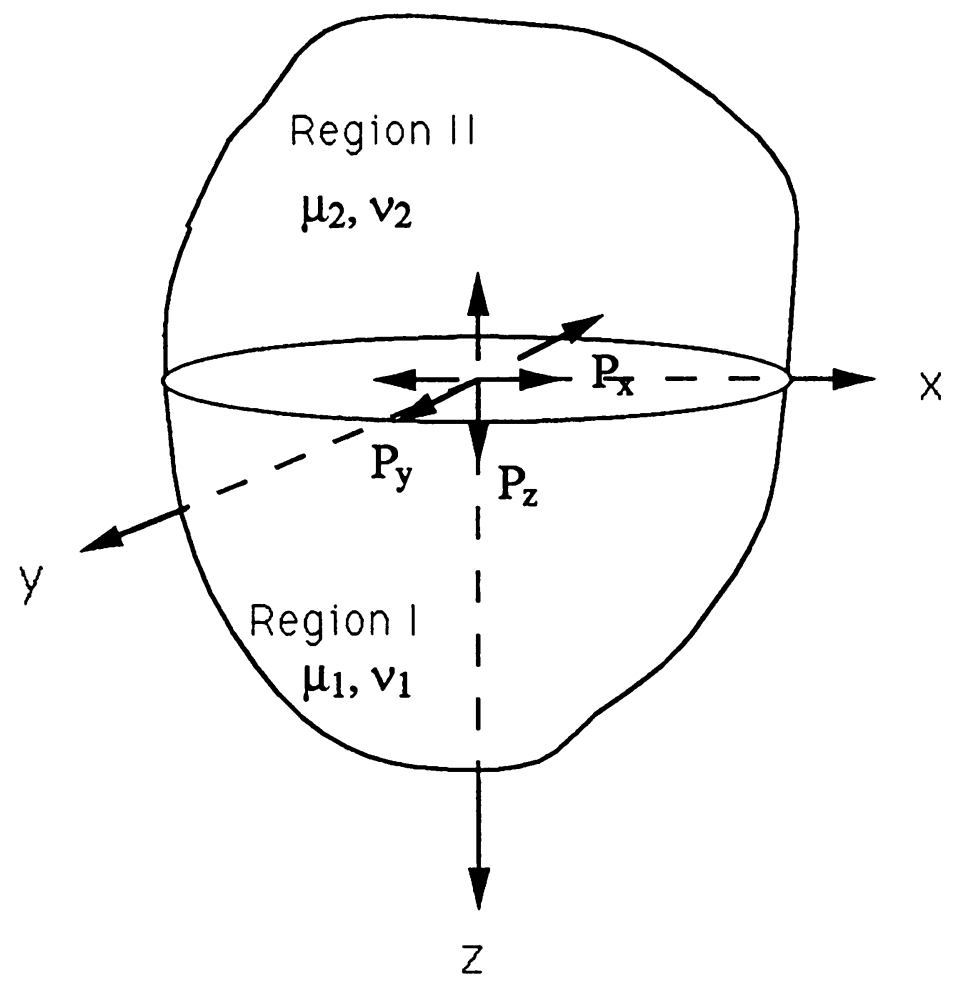

FIG. 1. A center of dilatation applied at the interface of two materials

obtain six displacement fields that are uniquely defined at the interface. Since for every displacement field there are three displacement components, therefore in total there exist 18 uniquely defined displacement components. However, these displacement components are not independent since the following relationships hold between the gradients of the displacement:

$$
\begin{aligned}
& \frac{\partial u_{y}^{\left(P_{x}\right)}}{\partial x}=\frac{\partial u_{x}^{\left(P_{y}\right)}}{\partial x}, \quad \frac{\partial u_{y}^{\left(P_{x}\right)}}{\partial y}=\frac{\partial u_{x}^{\left(P_{y}\right)}}{\partial y}, \quad \frac{\partial u_{z}^{\left(P_{x}\right)}}{\partial y}=\frac{\partial u_{z}^{\left(P_{y}\right)}}{\partial x}, \quad \frac{\partial u_{x}^{\left(P_{z}\right)}}{\partial y}=\frac{\partial u_{y}^{\left(P_{z}\right)}}{\partial x} \\
& \frac{\partial u_{z}^{\left(P_{z}\right)}}{\partial x}=-\left(\frac{\partial u_{x}^{\left(P_{x}\right)}}{\partial x}+\frac{\partial u_{y}^{\left(P_{x}\right)}}{\partial y}\right)-\frac{A \kappa_{1}^{2}+B+2 \kappa_{1}}{(1+s)\left(1+\kappa_{1}\right)}\left(\frac{\partial u_{x}^{\left(P_{y}\right)}}{\partial y}-\frac{\partial u_{y}^{\left(P_{y}\right)}}{\partial x}\right) \\
& \frac{\partial u_{z}^{\left(P_{z}\right)}}{\partial y}=-\left(\frac{\partial u_{y}^{\left(P_{y}\right)}}{\partial y}+\frac{\partial u_{x}^{\left(P_{y}\right)}}{\partial x}\right)-\frac{A \kappa_{1}^{2}+B+2 \kappa_{1}}{(1+s)\left(1+\kappa_{1}\right)}\left(\frac{\partial u_{y}^{\left(P_{x}\right)}}{\partial x}-\frac{\partial u_{x}^{\left(P_{x}\right)}}{\partial y}\right) .
\end{aligned}
$$

The first four are due to Betti's reciprocity relations; the last two are properties of the point force solutions.

Thus the 18 displacement functions reduce to 12 independent displacement functions that are uniquely defined at the interface of two materials. In order to obtain them we proceed by differentiating in $x$ and $y$ the displacement fields due to the forces $P_{x}, P_{y}, P_{z}$ 
at an interface (Rongved (1955)) and using the Dundurs constants. These 12 functions are:

For region I:

$$
\begin{aligned}
& g_{1}^{I}=\frac{1}{\mu_{1}} \frac{Q}{4 \pi\left(\kappa_{1}+1\right)}\left\{\left[\left(1+\kappa_{1}\right)\left(\frac{\beta-\alpha}{1+\beta} \kappa_{1}+1\right)-\left(\frac{(\beta-\alpha) \kappa_{1}^{2}}{2(1+\beta)}+\frac{\alpha+\beta}{2(1-\beta)}\right)\right] \frac{x}{R^{3}}\right. \\
& -\left(\frac{\beta-\alpha}{1+\beta} \kappa_{1}+1\right) \frac{3 x y^{2}}{R^{5}}-\left(\frac{\beta-\alpha}{1+\beta} \kappa_{1}+1\right) \frac{3 x z^{2}}{R^{5}}-\left[\left(1+\frac{\kappa_{1}}{2}\right)\left(\frac{\beta-\alpha}{1+\beta} \kappa_{1}+1\right)\right. \\
& \left.+\frac{1}{2} \frac{1+\alpha}{\beta-1}-\left(1+\kappa_{1}\right)\left(\frac{2 \kappa_{1}(\beta-\alpha)+2+2 \beta}{\kappa_{1}(\beta-\alpha)+2+\alpha+\beta}-\frac{1}{2}\right)\right]\left(\frac{x}{R(R+z)^{2}}\right. \\
& \left.\left.-\frac{x y^{2}}{R^{3}(R+z)^{2}}-\frac{2 x y^{2}}{R^{2}(R+z)^{3}}\right)\right\} \\
& g_{2}^{I}=-\frac{1}{\mu_{1}} \frac{Q}{4 \pi\left(\kappa_{1}+1\right)}\left\{\left(\frac{\beta-\alpha}{1+\beta} \kappa_{1}+1\right) \frac{y}{R^{3}}-\left(\frac{\beta-\alpha}{1+\beta} \kappa_{1}+1\right) \frac{3 x^{2} y}{R^{5}}\right. \\
& -\left[\frac{1}{2} \frac{1+\alpha}{\beta-1}-\left(1+\kappa_{1}\right)\left(\frac{2 \kappa_{1}(\beta-\alpha)+2+2 \beta}{\kappa_{1}(\beta-\alpha)+2+\alpha+\beta}-\frac{1}{2}\right)\right. \\
& \left.\left.+\left(1+\frac{\kappa_{1}}{2}\right)\left(\frac{\beta-\alpha}{1+\beta} \kappa_{1}+1\right)\right]\left(\frac{y}{R(R+z)^{2}}-\frac{x^{2} y}{R^{3}(R+z)^{2}}-\frac{2 x^{2} y}{R^{2}(R+z)^{3}}\right)\right\} \\
& g_{3}^{I}=-\frac{1}{\mu_{1}} \frac{Q}{4 \pi\left(\kappa_{1}+1\right)}\left\{\left(\frac{\beta-\alpha}{1+\beta} \kappa_{1}+1\right) \frac{z}{R^{3}}-\left(\frac{\beta-\alpha}{1+\beta} \kappa_{1}+1\right) \frac{3 x^{2} z}{R^{5}}\right. \\
& \left.+\left(\frac{(\beta-\alpha) \kappa_{1}^{2}}{2(1+\beta)}+\frac{\alpha+\beta}{2(1-\beta)}\right)\left(\frac{1}{R(R+z)}-\frac{x^{2}}{R^{2}(R+z)^{2}}-\frac{2 x^{2}}{R^{3}(R+z)}\right)\right\} \\
& g_{4}^{I}=\frac{1}{\mu_{1}} \frac{Q}{4 \pi\left(\kappa_{1}+1\right)}\left\{\left[\frac{\beta-\alpha}{1+\beta} \kappa_{1}+1-\left(1+\kappa_{1}\right) \frac{2 \kappa_{1}(\beta-\alpha)+2+2 \beta}{\kappa_{1}(\beta-\alpha)+2+\alpha+\beta}\right] \frac{y}{R^{3}}\right. \\
& -\left(\frac{\beta-\alpha}{1+\beta} \kappa_{1}+1\right) \frac{3 x^{2} y}{R^{5}}-\left[\frac{1}{2} \frac{1+\alpha}{\beta-1}-\left(1+\kappa_{1}\right)\left(\frac{2 \kappa_{1}(\beta-\alpha)+2+2 \beta}{\kappa_{1}(\beta-\alpha)+2+\alpha+\beta}-\frac{1}{2}\right)\right. \\
& \left.\left.+\left(1+\frac{\kappa_{1}}{2}\right)\left(\frac{\beta-\alpha}{1+\beta} \kappa_{1}+1\right)\right]\left(\frac{y}{R(R+z)^{2}}-\frac{x^{2} y}{R^{3}(R+z)^{2}}-\frac{2 x^{2} y}{R^{2}(R+z)^{3}}\right)\right\} \\
& g_{5}^{I}=\frac{1}{\mu_{1}} \frac{Q}{4 \pi\left(\kappa_{1}+1\right)}\left\{\left(\frac{\beta-\alpha}{1+\beta} \kappa_{1}+1\right) \frac{x}{R^{3}}-\left(\frac{\beta-\alpha}{1+\beta} \kappa_{1}+1\right) \frac{3 x y^{2}}{R^{5}}\right. \\
& -\left[\frac{1}{2} \frac{1+\alpha}{\beta-1}-\left(1+\kappa_{1}\right)\left(\frac{2 \kappa_{1}(\beta-\alpha)+2+2 \beta}{\kappa_{1}(\beta-\alpha)+2+\alpha+\beta}-\frac{1}{2}\right)\right. \\
& \left.\left.+\left(1+\frac{\kappa_{1}}{2}\right)\left(\frac{\beta-\alpha}{1+\beta} \kappa_{1}+1\right)\right]\left(\frac{x}{R(R+z)^{2}}-\frac{x y^{2}}{R^{3}(R+z)^{2}}-\frac{2 x y^{2}}{R^{2}(R+z)^{3}}\right)\right\} \\
& g_{6}^{I}=\frac{1}{\mu_{1}} \frac{Q}{4 \pi\left(\kappa_{1}+1\right)}\left\{-\left(\frac{(\beta-\alpha) \kappa_{1}^{2}}{2(1+\beta)}+\frac{\alpha+\beta}{2(1-\beta)}\right)\left(\frac{x y}{R^{2}(R+z)^{2}}+\frac{x y}{R^{3}(R+z)}\right)\right. \\
& \left.-\left(\frac{\beta-\alpha}{1+\beta} \kappa_{1}+1\right) \frac{3 x y z}{R^{5}}\right\} \\
& g_{7}^{I}=\frac{1}{\mu_{1}} \frac{Q}{4 \pi\left(\kappa_{1}+1\right)}\left\{\left[\left(1+\kappa_{1}\right)\left(\frac{\beta-\alpha}{1+\beta} \kappa_{1}+1\right)-\left(\frac{(\beta-\alpha) \kappa_{1}^{2}}{2(1+\beta)}+\frac{\alpha+\beta}{2(1-\beta)}\right)\right] \frac{y}{R^{3}}\right.
\end{aligned}
$$




$$
\begin{aligned}
& -\left(\frac{\beta-\alpha}{1+\beta} \kappa_{1}+1\right) \frac{3 x^{2} y}{R^{5}}-\left(\frac{\beta-\alpha}{1+\beta} \kappa_{1}+1\right) \frac{3 y z^{2}}{R^{5}}-\left[\left(1+\frac{\kappa_{1}}{2}\right)\left(\frac{\beta-\alpha}{1+\beta} \kappa_{1}+1\right)\right. \\
& \left.+\frac{1}{2} \frac{1+\alpha}{\beta-1}-\left(1+\kappa_{1}\right)\left(\frac{2 \kappa_{1}(\beta-\alpha)+2+2 \beta}{\kappa_{1}(\beta-\alpha)+2+\alpha+\beta}-\frac{1}{2}\right)\right]\left(\frac{y}{R(R+z)^{2}}\right. \\
& \left.\left.-\frac{x^{2} y}{R^{3}(R+z)^{2}}-\frac{2 x^{2} y}{R^{2}(R+z)^{3}}\right)\right\} \\
& g_{8}^{I}=-\frac{1}{\mu_{1}} \frac{Q}{4 \pi\left(\kappa_{1}+1\right)}\left\{\left(\frac{\beta-\alpha}{1+\beta} \kappa_{1}+1+\frac{(\beta-\alpha) \kappa_{1}^{2}}{2(1+\beta)}+\frac{\alpha+\beta}{2(1-\beta)}\right) \frac{z}{R^{3}}\right. \\
& -\left(\frac{\beta-\alpha}{1+\beta} \kappa_{1}+1\right) \frac{3 y^{2} z}{R^{5}}+\left(\frac{(\beta-\alpha) \kappa_{1}^{2}}{2(1+\beta)}+\frac{\alpha+\beta}{2(1-\beta)}\right)\left(\frac{1}{R(R+z)}\right. \\
& \left.\left.-\frac{x^{2}}{R^{2}(R+z)^{2}}-\frac{2 x^{2}}{R^{3}(R+z)}\right)\right\} \\
& g_{9}^{I}=\frac{1}{\mu_{1}} \frac{Q}{4 \pi\left(\kappa_{1}+1\right)}\left\{\left[\frac{\beta-\alpha}{1+\beta} \kappa_{1}+1-\left(1+\kappa_{1}\right) \frac{2 \kappa_{1}(\beta-\alpha)+2+2 \beta}{\kappa_{1}(\beta-\alpha)+2+\alpha+\beta}\right] \frac{x}{R^{3}}\right. \\
& -\left(\frac{\beta-\alpha}{1+\beta} \kappa_{1}+1\right) \frac{3 x y^{2}}{R^{5}}-\left[\left(1+\frac{\kappa_{1}}{2}\right)\left(\frac{\beta-\alpha}{1+\beta} \kappa_{1}+1\right)+\frac{1}{2} \frac{1+\alpha}{\beta-1}\right. \\
& \left.-\left(1+\kappa_{1}\right)\left(\frac{2 \kappa_{1}(\beta-\alpha)+2+2 \beta}{\kappa_{1}(\beta-\alpha)+2+\alpha+\beta}-\frac{1}{2}\right)\right]\left(\frac{x}{R(R+z)^{2}}-\frac{x y^{2}}{R^{3}(R+z)^{2}}\right. \\
& \left.\left.-\frac{2 x y^{2}}{R^{2}(R+z)^{3}}\right)\right\} \\
& g_{10}^{I}=\frac{1}{\mu_{1}} \frac{Q}{4 \pi\left(\kappa_{1}+1\right)}\left\{\left(\frac{\beta-\alpha}{1+\beta} \kappa_{1}+1\right) \frac{z}{R^{3}}-\left(\frac{\beta-\alpha}{1+\beta} \kappa_{1}+1\right) \frac{3 x^{2} z}{R^{5}}\right. \\
& \left.-\left(\frac{(\beta-\alpha) \kappa_{1}^{2}}{2(1+\beta)}+\frac{\alpha+\beta}{2(1-\beta)}\right)\left(\frac{1}{R(R+z)}-\frac{x^{2}}{R^{2}(R+z)^{2}}-\frac{2 x^{2}}{R^{3}(R+z)}\right)\right\} \\
& g_{11}^{I}=\frac{1}{\mu_{1}} \frac{Q}{4 \pi\left(\kappa_{1}+1\right)}\left\{\left(\frac{(\beta-\alpha) \kappa_{1}^{2}}{2(1+\beta)}+\frac{\alpha+\beta}{2(1-\beta)}\right)\left(\frac{x y}{R^{2}(R+z)^{2}}+\frac{x y}{R^{3}(R+z)}\right)\right. \\
& \left.-\left(\frac{\beta-\alpha}{1+\beta} \kappa_{1}+1\right) \frac{3 x y z}{R^{5}}\right\} \\
& g_{12}^{I}=\frac{1}{\mu_{1}} \frac{Q}{4 \pi\left(\kappa_{1}+1\right)}\left\{\left[\frac{\beta-\alpha}{1+\beta} \kappa_{1}+1-\left(\frac{(\beta-\alpha) \kappa_{1}^{2}}{2(1+\beta)}+\frac{\alpha+\beta}{2(1-\beta)}\right)\right] \frac{z}{R^{3}}\right. \\
& -\left(\frac{\beta-\alpha}{1+\beta} \kappa_{1}+1\right) \frac{3 y^{2} z}{R^{5}}+\left(\frac{(\beta-\alpha) \kappa_{1}^{2}}{2(1+\beta)}+\frac{\alpha+\beta}{2(1-\beta)}\right)\left(\frac{1}{R(R+z)}\right. \\
& \left.\left.-\frac{x^{2}}{R^{2}(R+z)^{2}}-\frac{2 x^{2}}{R^{3}(R+z)}\right)\right\} \text {. }
\end{aligned}
$$

To obtain the expression for region II, according to Dundurs and Markenscoff (1989), we let

$$
\begin{gathered}
\alpha \rightarrow-\alpha, \quad \beta \rightarrow-\beta, \quad \kappa_{1} \rightarrow \kappa_{2}, \quad \kappa_{2} \rightarrow \kappa_{1}, \quad \mu_{1} \rightarrow \mu_{2} \\
z \rightarrow-z, \quad g_{3} \rightarrow-g_{3}, \quad g_{6} \rightarrow-g_{6}, \quad g_{8} \rightarrow-g_{8} \\
g_{10} \rightarrow-g_{10}, \quad g_{11} \rightarrow-g_{11}, \quad g_{12} \rightarrow-g_{12}
\end{gathered}
$$


where

$$
\alpha=\frac{\Gamma\left(\kappa_{1}+1\right)-\left(\kappa_{2}+1\right)}{\Gamma\left(\kappa_{1}+1\right)+\kappa_{2}+1}, \quad \beta=\frac{\Gamma\left(\kappa_{1}-1\right)-\left(\kappa_{2}-1\right)}{\Gamma\left(\kappa_{1}+1\right)+\kappa_{2}+1}
$$

are the Dundurs' constants.

Considering these 12 independent displacement functions, there are only 12 independent terms involved. They are

$$
\begin{gathered}
\frac{x}{R^{3}}, \frac{y}{R^{3}}, \frac{z}{R^{3}}, \frac{x z^{2}}{R^{5}}, \frac{x^{2} z}{R^{5}}, \quad \frac{y z^{2}}{R^{5}}, \frac{y^{2} z}{R^{5}}, \frac{x y z}{R^{5}}, \\
\frac{1}{R(R+z)}-\frac{x^{2}}{R^{2}(R+z)^{2}}-\frac{x^{2}}{R^{3}(R+z)}, \frac{x y}{R^{2}(R+z)^{2}}+\frac{x y}{R^{3}(R+z)}, \\
\left(\frac{\beta-\alpha}{1+\beta} \kappa_{1}+1\right) \frac{3 x y^{2}}{R^{5}}+\left[\left(1+\frac{\kappa_{1}}{2}\right)\left(\frac{\beta-\alpha}{1+\beta} \kappa_{1}+1\right)-\left(1+\kappa_{1}\right)\left(\frac{2 \kappa_{1}(\beta-\alpha)+2+2 \beta}{\kappa_{1}(\beta-\alpha)+2+\alpha+\beta}\right.\right. \\
\left.\left.-\frac{1}{2}\right)+\frac{1}{2} \frac{1+\alpha}{\beta-1}\right]\left(\frac{x}{R(R+z)^{2}}-\frac{2 x y^{2}}{R^{2}(R+z)^{3}}-\frac{x y^{2}}{R^{3}(R+z)^{2}}\right), \quad(3) \\
\left(\frac{\beta-\alpha}{1+\beta} \kappa_{1}+1\right) \frac{3 x^{2} y}{R^{5}}+\left[\left(1+\frac{\kappa_{1}}{2}\right)\left(\frac{\beta-\alpha}{1+\beta} \kappa_{1}+1\right)-\left(1+\kappa_{1}\right)\left(\frac{2 \kappa_{1}(\beta-\alpha)+2+2 \beta}{\kappa_{1}(\beta-\alpha)+2+\alpha+\beta}\right.\right. \\
\left.\left.-\frac{1}{2}\right)+\frac{1}{2} \frac{1+\alpha}{\beta-1}\right]\left(\frac{y}{R(R+z)^{2}}-\frac{2 x^{2} y}{R^{2}(R+z)^{3}}-\frac{x^{2} y}{R^{3}(R+z)^{2}}\right) .
\end{gathered}
$$

III. Doublets of forces at an interface. The limits of all force doublets approaching an interface from either side have been obtained in (Ye, 1992). We will show here that all the limits of these doublets as they approach the interface can be presented as a linear combination of the previously obtained 12 independent displacement functions (2), and hence (3).

- Double force in $x$-direction with moment about $y$-direction

Case (1). The doublet approaches the interface from material I:

$$
\begin{aligned}
u_{x}^{I}= & \frac{1}{2 a_{1} a_{2}}\left\{\left(-a_{1} a_{6}-a_{1} a_{7}-a_{2} a_{7}+a_{1} a_{8}+a_{1} a_{9}\right) g_{10}^{I}+\left(-a_{1} a_{6}-a_{1} a_{7}\right) g_{12}^{I}\right. \\
& \left.-\left(a_{1} a_{6}+a_{1} a_{7}-a_{2} a_{7}-a_{1} a_{8}-a_{1} a_{9}\right) g_{3}^{I}-\left(a_{1} a_{6}+a_{1} a_{7}\right) g_{8}^{I}\right\}, \\
u_{y}^{I}= & \frac{1}{2 a_{1} a_{2}}\left\{\left(-a_{2} a_{7}+a_{1} a_{8}+a_{1} a_{9}\right) g_{11}^{I}-\left(a_{2} a_{7}+a_{1} a_{8}+a_{1} a_{9}\right) g_{6}^{I}\right\}, \\
u_{z}^{I}= & \frac{1}{a_{1}\left(a_{5}-a_{1}\right)}\left\{\left(a_{1} a_{7}-a_{5} a_{7}\right) g_{1}^{I}+\left(a_{1}^{2}+a_{1} a_{2}-a_{1} a_{7}\right.\right. \\
& \left.\left.-a_{3} a_{7}+a_{5} a_{7}\right) g_{5}^{I}+\left(a_{3} a_{7}-a_{1} a_{2}-a_{1}^{2}\right) g_{9}^{I}\right\} .
\end{aligned}
$$


Case (2). The doublet approaches the interface from material II:

$$
\begin{aligned}
u_{x}^{I}= & \frac{1}{2 a_{1} a_{2}}\left\{\left(a_{2} a_{12}+a_{1} a_{13}+a_{1} a_{14}-a_{1} a_{15}\right) g_{10}^{I}+a_{1} a_{13} g_{12}^{I}\right. \\
& \left.-\left(a_{2} a_{12}-a_{1} a_{13}-a_{1} a_{14}+a_{1} a_{15}\right) g_{3}^{I}+a_{1} a_{13} g_{8}^{I}\right\} \\
u_{y}^{I}= & \frac{1}{2 a_{1} a_{2}}\left\{\left(a_{2} a_{12}+a_{1} a_{14}-a_{1} a_{15}\right) g_{11}^{I}+\left(a_{2} a_{12}-a_{1} a_{14}+a_{1} a_{15}\right) g_{6}^{I}\right\}, \\
u_{z}^{I}= & \frac{1}{a_{1}\left(a_{5}-a_{1}\right)}\left\{\left(a_{5} a_{12}-a_{1} a_{12}\right) g_{1}^{I}+\left(a_{1} a_{12}+a_{3} a_{12}-a_{5} a_{12}-a_{1} a_{16}-a_{1} a_{17}\right) g_{5}^{I}\right. \\
& \left.+\left(a_{1} a_{16}+a_{1} a_{17}-a_{3} a_{12}\right) g_{9}^{I}\right\} .
\end{aligned}
$$

- Double force in $y$-direction:

$$
u_{x}^{I}=g_{5}^{I}
$$

- Double force in $y$-direction with moment about $z$-direction:

$$
\begin{aligned}
& u_{x}^{I}=g_{2}^{I} \\
& u_{z}^{I}=g_{6}^{I}
\end{aligned}
$$

- Double force in $y$-direction with moment about $x$-direction

Case (1). The doublet approaches the interface from material I:

$$
\begin{aligned}
u_{x}^{I}= & \frac{1}{2 a_{1} a_{2}}\left\{\left(a_{1} a_{8}-a_{2} a_{7}\right) g_{11}^{I}-\left(a_{2} a_{7}+a_{1} a_{8}+a_{1} a_{9}\right) g_{6}^{I}\right\}, \\
u_{y}^{I}= & \frac{1}{2 a_{1} a_{2}}\left\{-\left(a_{1} a_{6}+a_{1} a_{7}\right) g_{10}^{I}+\left(a_{1} a_{9}+a_{1} a_{8}-a_{1} a_{2}-a_{1} a_{7}-a_{2} a_{7}\right) g_{12}^{I}\right. \\
& \left.-\left(a_{1} a_{6}+a_{1} a_{7}\right) g_{3}^{I}+\left(a_{1} a_{9}+a_{1} a_{8}+a_{2} a_{7}-a_{1} a_{7}-a_{1} a_{6}\right) g_{8}^{I}\right\}, \\
u_{z}^{I}= & \frac{1}{a_{1}\left(a_{5}-a_{1}\right)}\left\{\left(-a_{1}^{2}-a_{1} a_{2}+a_{1} a_{7}+a_{3} a_{7}-a_{5} a_{7}\right) g_{2}^{I}+\left(a_{3} a_{7}-a_{1}^{2}-a_{1} a_{2}\right) g_{4}^{I}\right. \\
& \left.+\left(a_{1} a_{7}-a_{5} a_{7}\right) g_{7}^{I}\right\} .
\end{aligned}
$$

Case (2). The doublet approaches the interface from material II:

$$
\begin{aligned}
u_{x}^{I}= & \frac{1}{2 a_{1} a_{2}}\left\{\left(a_{2} a_{12}+a_{1} a_{14}-a_{1} a_{15}\right) g_{11}^{I}+\left(a_{2} a_{12}-a_{1} a_{14}+a_{1} a_{15}\right) g_{6}^{I}\right\} \\
u_{y}^{I}= & \frac{1}{2 a_{1} a_{2}}\left\{a_{1} a_{13} g_{10}^{I}+\left(a_{1} a_{13}+a_{2} a_{12}+a_{1} a_{14}-a_{1} a_{15}\right) g_{12}^{I}+a_{1} a_{13} g_{3}^{I}\right. \\
& \left.+\left(a_{1} a_{13}+a_{1} a_{14}-a_{1} a_{15}-a_{2} a_{12}\right) g_{8}^{I}\right\} \\
u_{z}^{I}= & \frac{1}{a_{1}\left(a_{5}-a_{1}\right)}\left\{\left(a_{1} a_{17}+a_{1} a_{16}+a_{5} a_{12}-a_{3} a_{12}-a_{1} a_{12}\right) g_{2}^{I}\right. \\
& \left.+\left(a_{1} a_{17}+a_{1} a_{16}-a_{3} a_{12}\right) g_{4}^{I}+\left(a_{5} a_{12}-a_{1} a_{12}\right) g_{7}^{I}\right\} .
\end{aligned}
$$

- Double force in $z$-direction 
Case (1). The doublet approaches the interface from material I:

$$
\begin{aligned}
u_{x}^{I}= & \frac{1}{a_{1}\left(a_{5}-a_{1}\right)}\left\{\left(a_{1} a_{10}-a_{10} a_{5}\right) g_{1}^{I}+\left(a_{1}^{2}-a_{1} a_{10}-a_{1} a_{2}-a_{10} a_{3}+a_{10} a_{5}\right) g_{5}^{I}\right. \\
& \left.+\left(a_{10} a_{3}+a_{1} a_{2}-a_{1}^{2}\right) g_{9}^{I}\right\}, \\
u_{y}^{I}= & \frac{1}{a_{1}\left(a_{5}-a_{1}\right)}\left\{\left(a_{1} a_{10}+a_{1} a_{2}-a_{1}^{2}+a_{10} a_{3}-a_{10} a_{5}\right) g_{2}^{I}\right. \\
& \left.-\left(a_{10} a_{3}+a_{1} a_{2}-a_{1}^{2}\right) g_{4}^{I}+\left(a_{1} a_{10}-a_{4} a_{5}\right) g_{7}^{I}\right\}, \\
u_{z}^{I}= & \frac{1}{2 a_{1} a_{2}}\left\{\left(a_{1} a_{11}-a_{1} a_{7}+a_{2} a_{7}\right) g_{10}^{I}+\left(a_{1} a_{11}-a_{1} a_{7}+a_{2} a_{7}\right) g_{12}^{I}\right. \\
& \left.+\left(a_{1} a_{11}-a_{1} a_{7}-a_{2} a_{7}\right) g_{3}^{I}+\left(a_{1} a_{11}-a_{1} a_{7}-a_{2} a_{7}\right) g_{8}^{I}\right\} .
\end{aligned}
$$

Case (2). The doublet approaches the interface from material II:

$$
\begin{aligned}
u_{x}^{I}= & \frac{1}{a_{1}\left(a_{5}-a_{1}\right)}\left\{\left(a_{5} a_{12}-a_{1} a_{12}\right) g_{1}^{I}+\left(a_{1} a_{12}+a_{3} a_{12}-a_{5} a_{12}-a_{1} a_{16}+a_{1} a_{17}\right) g_{5}^{I}\right. \\
& \left.+\left(a_{1} a_{16}-a_{1} a_{17}-a_{3} a_{12}\right) g_{9}^{I}\right\}, \\
u_{y}^{I}= & \frac{1}{a_{1}\left(a_{5}-a_{1}\right)}\left\{\left(a_{1} a_{16}+a_{5} a_{12}-a_{1} a_{12}-a_{3} a_{12}-a_{1} a_{17}\right) g_{2}^{I}\right. \\
& \left.-\left(a_{1} a_{16}-a_{3} a_{12}-a_{1} a_{17}\right) g_{4}^{I}+\left(a_{5} a_{12}-a_{1} a_{12}\right) g_{7}^{I}\right\}, \\
u_{z}^{I}= & \frac{1}{2 a_{1} a_{2}}\left\{\left(a_{1} a_{18}-a_{1} a_{16}-a_{2} a_{12}\right) g_{10}^{I}+\left(a_{1} a_{18}-a_{1} a_{16}-a_{2} a_{12}\right) g_{12}^{I}\right. \\
& \left.+\left(a_{1} a_{18}-a_{1} a_{16}+a_{2} a_{12}\right) g_{3}^{I}+\left(a_{1} a_{18}-a_{1} a_{16}+a_{2} a_{12}\right) g_{8}^{I}\right\} .
\end{aligned}
$$

- Double force in $z$-direction with moment about $y$-direction:

$$
\begin{aligned}
& u_{y}^{I}=g_{11}^{I}, \\
& u_{z}^{I}=\frac{1}{a_{5}-a_{1}}\left\{\left(a_{5}-a_{1}\right) g_{1}^{I}+\left(a_{1}+2 a_{3}-a_{5}\right) g_{5}^{I}-2 a_{3} g_{9}^{I}\right\} .
\end{aligned}
$$

- Double force in $z$-direction with moment about $x$-direction:

$$
u_{z}^{I}=\frac{1}{a_{5}-a_{1}}\left\{\left(a_{5}-a_{1}\right) g_{7}^{I}-\left(a_{1}+2 a_{3}-a_{5}\right) g_{2}^{I}-2 a_{3} g_{4}^{I}\right\}
$$

where

$$
\begin{aligned}
& a_{1}=\frac{\beta-\alpha}{1+\beta} \kappa_{1}+1 \\
& a_{2}=\frac{(\beta-\alpha) \kappa_{1}^{2}}{2(1+\beta)}+\frac{\alpha+\beta}{2(1-\beta)}, \\
& a_{3}=\kappa_{1}\left(\frac{\beta-\alpha}{1+\beta} \kappa_{1}+1\right)-\left(\frac{(\beta-\alpha) \kappa_{1}^{2}}{2(1+\beta)}+\frac{\alpha+\beta}{2(1-\beta)}\right), \\
& a_{4}=\left(1+\frac{\kappa_{1}}{2}\right)\left(\frac{\beta-\alpha}{1+\beta} \kappa_{1}+1\right)-\left(1+\kappa_{1}\right)\left(\frac{2 \kappa_{1}(\beta-\alpha)+2+2 \beta}{\kappa_{1}(\beta-\alpha)+2+\alpha+\beta}-\frac{1}{2}\right)+\frac{1}{2} \frac{1+\alpha}{\beta-1}, \\
& a_{5}=\frac{\beta-\alpha}{1+\beta} \kappa_{1}+1-\left(1+\kappa_{1}\right) \frac{2 \kappa_{1}(\beta-\alpha)+2+2 \beta}{\kappa_{1}(\beta-\alpha)+2+\alpha+\beta}, \\
& a_{6}=\frac{\beta-\alpha}{1+\beta} \kappa_{1}+1-\frac{2 \kappa_{1}(\beta-\alpha)+2+2 \beta}{\kappa_{1}(\beta-\alpha)+2+\alpha+\beta}-\frac{2 \alpha-a \beta}{1+\beta}+\frac{2 \kappa_{1}\left[\kappa_{2}(\alpha-\beta)+1-\beta\right]}{\kappa_{2}(\alpha-\beta)+2-\alpha-\beta},
\end{aligned}
$$




$$
\begin{aligned}
& a_{7}= \frac{2+2 \alpha}{1+\beta}-\left(\frac{\beta-\alpha}{1+\beta} \kappa_{1}+1\right) \\
& a_{8}= \frac{\beta-\alpha}{1+\beta} \kappa_{1}+1-\frac{2 \kappa_{1}(\beta-\alpha)+2+2 \beta}{\kappa_{1}(\beta-\alpha)+2+\alpha+\beta} \\
& a_{9}= \frac{\kappa_{1}}{2}\left(\frac{\beta-\alpha}{1+\beta} \kappa_{1}+1\right)-\kappa_{1} \frac{2 \kappa_{1}(\beta-\alpha)+2+2 \beta}{\kappa_{1}(\beta-\alpha)+2+\alpha+\beta}+\frac{1}{2}\left(1+\kappa_{1}+\frac{1+\alpha}{\beta-1}\right) \\
& a_{10}= \frac{2-2 \alpha+4 \beta}{1-\beta}-\left(\frac{\beta-\alpha}{1+\beta} \kappa_{1}+1\right) \\
& a_{11}= \kappa_{1}\left(\frac{\beta-\alpha}{1+\beta} \kappa_{1}+1\right)-\left(\frac{(\beta-\alpha) \kappa_{1}^{2}}{2(1+\beta)}+\frac{\alpha+\beta}{2(1-\beta)}\right)+2\left(1-\kappa_{1}\right) \frac{1-\alpha+2 \beta}{1+\beta} \\
& a_{12}= \frac{1-\alpha}{1+\beta}, \\
& a_{13}= \frac{2(1-\alpha)\left(1+\kappa_{2}\right)}{\kappa_{2}(\alpha-\beta)+2-\alpha-\beta}, \\
& a_{14}= \frac{1-\alpha}{1-\beta}-\frac{2(1-\alpha)\left(1+\kappa_{2}\right)}{\left[\kappa_{2}(\alpha-\beta)+2-\alpha-\beta\right]\left(1+\kappa_{1}\right)}, \\
& a_{15}= \frac{\kappa_{2}(\alpha-1)}{2(1-\beta)}+\frac{\kappa_{1}(\alpha-1)}{2(1+\beta)}+\frac{2 \kappa_{1}(1-\alpha)\left(1+\kappa_{2}\right)}{\left[\kappa_{2}(\alpha-\beta)+2-\alpha-\beta\right]\left(1+\kappa_{1}\right)}, \\
& a_{16}= \frac{1-\alpha}{1-\beta}, \\
& a_{17}= \frac{1-\alpha}{2}\left(\frac{\kappa_{2}}{1-\beta}-\frac{\kappa_{1}}{1+\beta}\right) \\
& a_{1}\left(\frac{\kappa_{2}}{1-\beta}+\frac{\kappa_{1}}{1+\beta}\right) . \\
&=
\end{aligned}
$$

IV. Center of dilatation at a bimaterial interface. Since all nuclei of strain consist of combinations of force doublets and all the limits of the force doublets as they approach the interface can be expressed in terms of the twelve uniquely defined displacement components, then all nuclei of strain as they approach the interface will be a linear combination of these twelve uniquely defined functions. For example, for a center of dilatation that is not uniquely defined at an interface, we find below that the two limits of each of the two (continuous across the interface) displacement fields, as the interface is approached from material 1 or from material 2, are:

$$
\begin{aligned}
& u_{x}^{I}=\left(1-\frac{a_{10}}{a_{1}}\right) g_{1}^{I}+\left(1+\frac{a_{10}}{a_{1}}+\frac{a_{1}^{2}-a_{10} a_{3}-a_{1} a_{2}}{a_{1}\left(a_{5}-a_{1}\right)}\right) g_{5}^{I}+\frac{a_{10} a_{3}+a_{1} a_{2}-a_{1}^{2}}{a_{1}\left(a_{5}-a_{1}\right)} g_{9}^{I}, \\
& u_{y}^{I}=\left(1-\frac{a_{10}}{a_{1}}\right) g_{7}^{I}+\left(1-\frac{a_{10}}{a_{1}}-\frac{a_{1}^{2}-a_{10} a_{3}-a_{1} a_{2}}{a_{1}\left(a_{5}-a_{1}\right)}\right) g_{2}^{I}-\frac{a_{10} a_{3}+a_{1} a_{2}-a_{1}^{2}}{a_{1}\left(a_{5}-a_{1}\right)} g_{4}^{I}, \\
& u_{z}^{I}=\left(1+\frac{a_{1} a_{11}-a_{1} a_{7}-a_{2} a_{7}}{2 a_{1} a_{2}}\right)\left(g_{3}^{I}+g_{8}^{I}\right)+\frac{a_{1} a_{11}-a_{1} a_{7}+a_{2} a_{7}}{2 a_{1} a_{2}}\left(g_{10}^{I}+g_{12}^{I}\right) ;
\end{aligned}
$$


Case (2). The center of dilatation approaches the interface from material II:

$$
\begin{aligned}
& u_{x}^{I}=\left(1-\frac{a_{12}}{a_{1}}\right) g_{1}^{I}+\left(1+\frac{a_{12}}{a_{1}}+\frac{a_{16} a_{1}-a_{12} a_{3}-a_{1} a_{17}}{a_{1}\left(a_{5}-a_{1}\right)}\right) g_{5}^{I}+\frac{a_{12} a_{3}+a_{1} a_{17}-a_{1} a_{16}}{a_{1}\left(a_{5}-a_{1}\right)} g_{9}^{I}, \\
& u_{y}^{I}=\left(1-\frac{a_{12}}{a_{1}}\right) g_{7}^{I}+\left(1-\frac{a_{12}}{a_{1}}+\frac{a_{16} a_{1}-a_{12} a_{3}-a_{1} a_{17}}{a_{1}\left(a_{5}-a_{1}\right)}\right) g_{2}^{I}-\frac{a_{12} a_{3}+a_{1} a_{17}-a_{1} a_{16}}{a_{1}\left(a_{5}-a_{1}\right)} g_{4}^{I}, \\
& u_{z}^{I}=\left(1-\frac{a_{1} a_{18}-a_{1} a_{16}+a_{2} a_{12}}{2 a_{1} a_{2}}\right)\left(g_{3}^{I}+g_{8}^{I}\right)-\frac{a_{1} a_{18}-a_{1} a_{16}-a_{2} a_{12}}{2 a_{1} a_{2}}\left(g_{10}^{I}+g_{12}^{I}\right) .
\end{aligned}
$$

The limits of the center of dilatation approaching the interface from material I and II are different (and each one is continuous at the interface as required), but each one is a (different) linear combination of the twelve uniquely defined displacement functions at the interface.

In this paper, we find twelve independent displacement functions uniquely defined at the interface of two materials. Also, we show that the displacement fields of all the nuclei of strain as they approach the interface can be expressed as linear combinations of these functions. These unique quantities can be used as Green's functions for cracks, inclusions, and hydraulic fracture at 3-D interfaces.

Acknowledgment. The support of the National Science Foundation and the UCSD Academic Senate is gratefully acknowledged.

\section{REFERENCES}

[1] J. L. Carvalho and J. H. Curran, Two-dimensional Green's functions for elastic bi-materials, J. Appl. Mech. ASME 59, 321-327 (1992)

[2] J. Dundurs and M. Hetényi, Transmission of force between two semi-infinite solids, J. Appl. Mech. ASME 32, 671-674 (1965)

[3] J. Dundurs and X. Markenscoff, The Sternberg-Koiter conclusion and other anomalies of the concentrated couple, J. Appl. Mech. ASME 56, 240-245 (1989)

[4] X. Markenscoff, J. Dundurs, and W. Ye, Elastic singularities at bimaterial interfaces, AMD, Vol. 148, Defects and Anelasticity in the Characterization of Crystalline Solids, ASME, 1992, pp. 93-96

[5] R. D. Mindlin and D. H. Cheng, Nuclei of strain in the semi-infinite solid, J. Appl. Phys. 21, 926-930 (1950)

[6] L. Rongved, Force interior to one of two joined semi-infinite solids, In Proc. First Midwestern Conf. on Solid Mech., University of Illinois, Urbana, Illinois, 1955, pp. 55-59

[7] S. Vijayakumar and D. E. Cormack, Green's functions for the biharmonic equation: Bounded elastic media. SIAM J. Appl. Math. 47, No. 5, 982-997 (1987)

[8] S. Vijayakumar and D. E. Cormack, Nuclei of strain for bi-material elastic media with sliding interface, Journal of Elasticity 17, 285-290 (1987)

[9] W. Ye, Nuclei of strain at bimaterial interfaces, Master's thesis, University of California, San Diego, 1992

[10] H. Y. Yu and S. C. Sanday, Elastic fields in joined half-spaces due to nuclei of strain, Proc. Royal Soc. London A 434, 503-519 (1991) 